\title{
Development of Automated Analysis System for Model Plane Engine Using Fuzzy Knowledge Processing
}

\author{
Joon-Seong Lee and Shin-Pyo Lee
}

Division of Electronic \& Mechanical Engineering, Kyonggi University, Suwon

\begin{abstract}
This paper describes a new automated analysis system for model plane engine. An automatic finite element (FE) mesh generation technique, which is based on the fuzzy knowledge processing and computational geometry technique, is incorporated into the system, together with one of commercial FE analysis codes, ANSYS, and one of commercial solid modelers, Designbase. The system allows a geometry model of concern to be automatically converted to different FE models, depending on physical phenomena of plane engine to be analyzed, i.e. deformation analysis, thermal analysis and so on. The FE models are then automatically analyzed by the FE analysis code. Among a whole process of analysis, the definition of a geometry model, the designation of local node patterns, the assignment of material properties and boundary conditions onto the geometry model are only the interactive processes to be done by a user. The interactive operations can be processed in a few minutes. The other processes which are time consuming and labour-intensive in conventional CAE systems are fully automatically performed in a personal computer environment. The proposed analysis system is successfully applied to evaluate a model plane engine.
\end{abstract}

Key Words : Fuzzy Knowledge Processing, Computational Geometry, Finite Element Analysis, Model Plane

\section{Introduction}

The design of structural component is an iterative process in which the aim is to achieve a structure which has adequate strength and stiffness, and is both practical and economical to manufacture : that is, in a some sense, an optimum design. The design procedure can take a very long time if approached conventionally, and it is unlikely that components will in fact be optimized in detail against all important criteria.

The use of an automated analysis system, involving FE codes together with CAD systems and FE pre-and post processors, has provided an important step towards shortening the design process and structural optimization. Of course, to do analysis and design work various general purpose programs such as Pro/Engineer ing, I-DEAS, ANSYS and so on have been used. Lsing these programs, however, conventional analyses of practical structures are still labour intensive and are not easy for ordinary designers and engineers to perform. Furthermore, it is difficult for them to find a satis factory or optimized solution of practical structures utilizing such conventional tools. A lot of trial and error evaluations are indispensable.

The finite element method (FEM) is now widely used

접수일자 : 2001년 12월 20일

완료일자 : 2002년 3월 20일

This research was supported by the Kyonggi University research grants in 2000 . for the analysis of many engineering problems involving static, dynamic, and thermal stressing of structures. The main reason for this is its high capability of dealing with boundary-value problems in arbitrarily shaped domains. On the other hand, a mesh used influences computational accuracy as well as time so significantly that the mesh generation process is as much important as the FEM analysis itself. Especially, in such large scale nonlinear FEM analyses that approach the limitation of computational capability of so called supercomputers, it is highly demanded to optimize the distribution of mesh size under the condition of limited total degrees of freedom.

Particularly, the mesh generation process, which influences computational accuracy as efficiency and whose fully automation is very difficult in three-dimensional cases, has become the most critical issue in a whole process of the $\mathrm{FE}$ analyses. In this respect, various researches $[1-5]$ have been performed on the development of automatic mesh generation techniques. However, the technique of finite elements is not often easy to use, because of the problem of formulating the necessary input data for a $\mathrm{FE}$ analysis program.

To efficiently support design processes of practical structures such as plane engine, the automatic FE mesh generator[6], which is based on the fuzzy knowledge processing and computational geometry techniques, is integrated with one of commercial $\mathrm{FE}$ analysis codes ANSYS[7] and one of commercial solid modelers Designbase[8].

This system is capable of providing the following facilities : 
(1) Definition of a geometry model, i.e. solid modeling including boolean operations such as union and intersection.

(2) Attachment of boundary conditions and material properties directly to parts of the geometry model.

(3) Ability to control node density.

(4) Facility for concentrating the mesh over any region.

(5) Fully automated mesh generation.

(6) Speedy node and element generation system which will lead to computational efficiency.

(7) Preparation of input data for analysis program.

(8) Various FE analyses such as electrostatic, stress and eigen value analyses.

(9) Visualization of analysis models and results

(10) The system is easy to use with minimum of input, and economical with respect to both computer time and manual effort.

Most part of the present system except the FE program is constructed on one of popular personal computers using the $\mathrm{C}^{++}$language.

\section{Outline of the System}

The developed FE analysis system allows designers to evaluate detailed physical behaviors of structures through some simple interactive operations to their geometry models. In other words, designers do not have to deal with mesh data when they operate the system. A flow of analyses using the system is shown in Fig. 1. Each subprocess will be described below.

\subsection{Definition of geometry model}

The advantage of Deginebase is that a side range of solid shapes from polyhedra to free-form surfaces can be designed in a unified manner. One of commercial geometric modelers, Deginebase[8] is employed for threedimensional solid structures. As an example, Fig. 2 shows a geometry model of three-dimensional solid structures. In these modelers, three-dimensional geometric data are stored as a tree structure of domainsurfaces (free-form surfaces such as Bezier or Gregory type surfaces)-edge (B-spline or Bezier type curves)vertices.

\subsection{Attachment of material properties and boundary conditions}

Material properties and boundary conditions are directly attached onto the geometry model by clicking the loops or edges that are parts of the geometric model using a mouse, and then by inputting actual values. The boundary conditions of the present system accept Direchlet's and Neumann's type. This system allows the user to enter mechanical loads in various forms for stress analysis. In a heat transfer analysis, the user can enter heat flux or temperature.

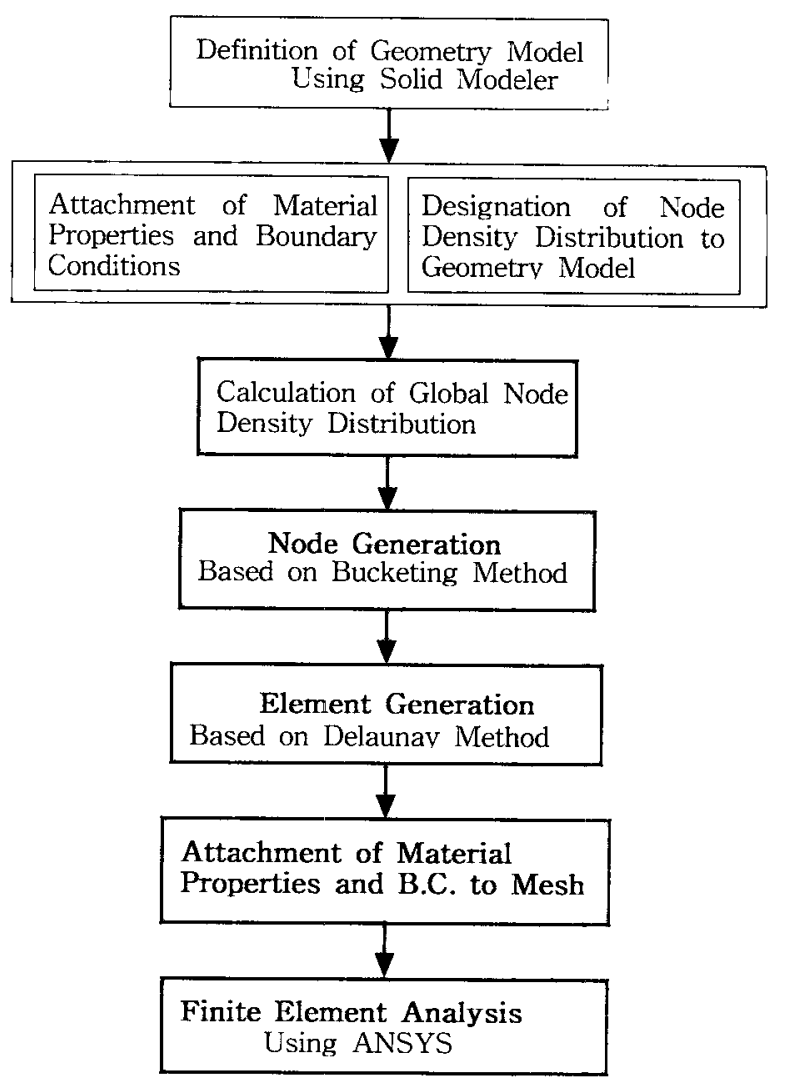

Fig. 1. Flow of analysis

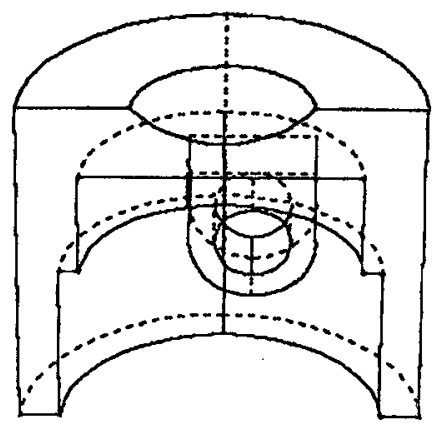

Fig. 2. Example of geometry model

\subsection{Designation of node density distribution}

In the present system, nodes are first generated, and then an FE mesh is built. In general, it is not so easy to well control element size for a complex geometry. Example of node density function is shown in Fig. 3. A node density distribution over a whole geometry model is constructed as follows. The present system stores several local node patterns such as the pattern suitable to well capture stress concentration, the pattern to subdivided a finite domain uniformly, and the pattern to subdivide a whole domain uniformly. An user selects some of those local node patterns, depending on their analysis purposes, and designates their relative importance and where to locate them. The process is illustrated in Fig. 4. For example, when either the crack or the hole exists solely in an infinite domain, the local 

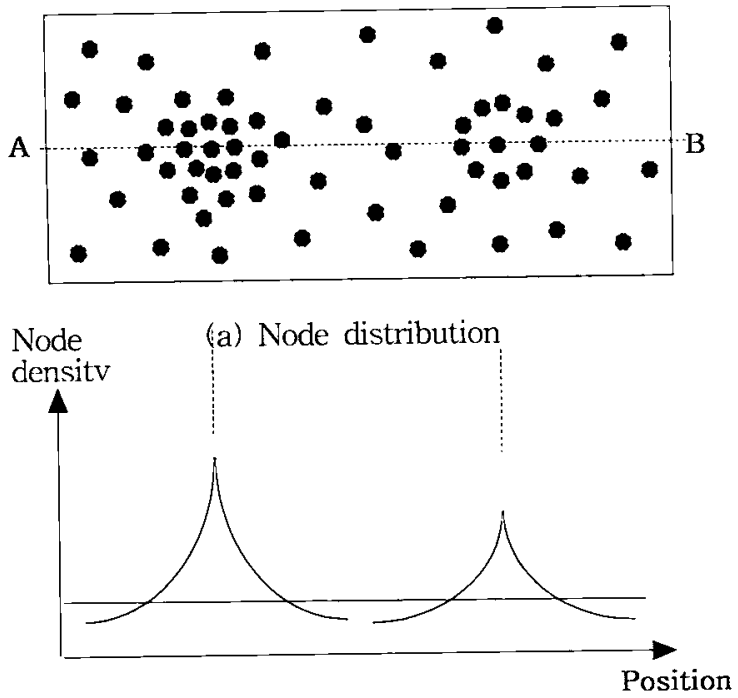

(b) Node density function along line $\mathrm{A}-\mathrm{B}$

Fig. 3. Example of node density function

node patterns may be regarded locally-optimum around the crack-tip or the hole. When these stress concentration sources exist closely to each other in the analysis domain, extra nodes have to be removed from the superposed region of both patterns. In the presented method, a global distribution of node density over the whole analysis domain is then automatically calculated through their superposition using the fuzzy knowledge processing[9,10]. In the conventional method, it is divided a single domain into sub-domains a prior : (a) a sub domain close to the crack tip and (b) that of the hole. However, the concept of "closenes" is in general so ambiguous that it is very defficult to define the subdomain. Thus, the concept of the fuzzy theory such as membership function is introduced here as follows.

Fig. 4(c) shows the membership functions corresponding to the node pattern around the hole and that of the crack tip respectively. For the purpose of simplicity, each membership function is given here to be a function of one dimensional location. In the Figs., the horizontal axis denotes the location, while the vertical axis denotes the value of the membership function, which indicates the magnitude of "closeness" or "membership" of the location to each stress concentration field. That is, a closer nodal location takes a larger value of membership function. Also, since finer node patterns are generally required to be placed near stress concentration fields, it is convenient to let the membership function correspond to a node density distribution. As shown in Fig. 4(d) and $4(e)$, the single analysis domain is automatically divided into two sub-domains $A$ and $B$ as a result of the fuzzy knowledge processing using membership functions. That is, the membership function with a larger value is employed through the comparison of values of both membership functions at each location. Both node patterns are placed in the sub-domain $A$ and $B$ as shown in Fig. 4(e). In other words, extra nodes are automatically removed from the superposed resion of both node patterns. When designers do not want any special meshing, they can adopt uniformly subdivided mesh.

(a)

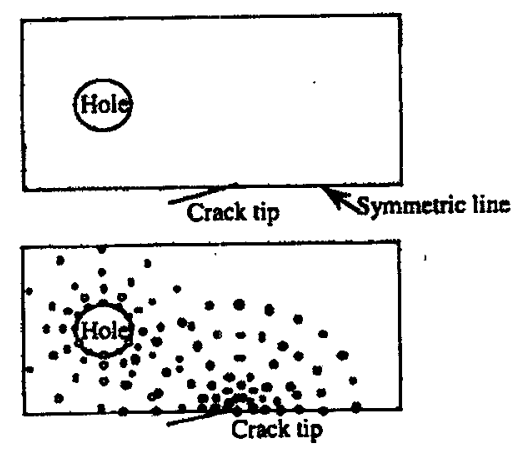

(c)

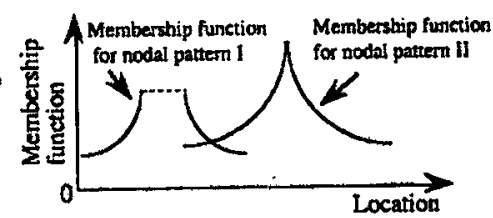

(d)

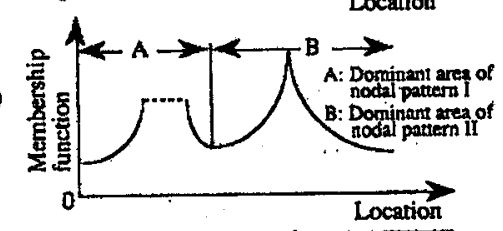

(c)
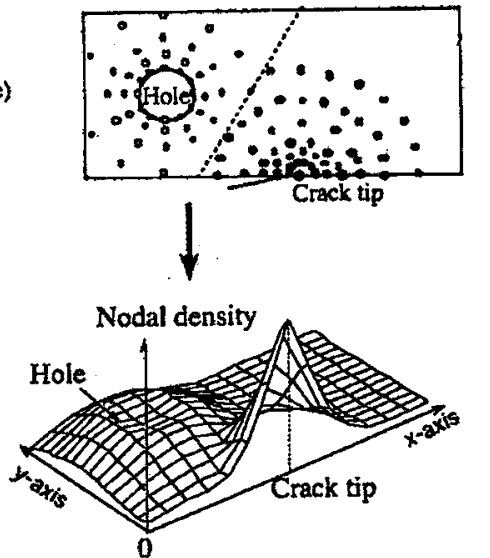

Fig. 4. Superposition of node patterns based on fuzzy knowledge processing

\subsection{Node generation}

Node generation is one of time consuming processes in automatic mesh generation. In the present study, the bucketing method[11] is adopted to generate nodes which satisfy the distribution of node density over a whole analysis domain.

At first, a number of candidate nodes with uniform spacing are prepared in one of buckets as shown in Fig. 5. The distance of two neighboring candidate nodes is set to be smaller than the minimum distance of nodes to be generated in the relevant bucket. Next, candidate nodes are pick up one by one, starting from the left- 
bottom corner of the bucket, and are put into the bucket. A candidate node is adopted as one of the final nodes when it satisfies the following two criteria :

(a) The candidate node is inside the analysis domain (IN/OLT check).

(b) The distance between the candidate node and the nearest node already generated in the bucket satisfies the node density at the point to some extent.

Practically, the criterion (a) is first examined bucket by bucket. As for buckets lying across the domain boundary, the criterion (a) is examined node by node. It should be noted here that the nodes already generated in the neighboring buckets have to be examined for the criterion (b) as well when a candidate node is possibly generated near the border of the relevant bucket. Thanks to the bucketing method, the number of examinations of the criterion (b) can be reduced significantly, and then a node generation speed is remained to be proportional to the total number of nodes. As for three-dimensional solid geometries, nodes are generated in the following order : vertices, edges, surfaces and domain.

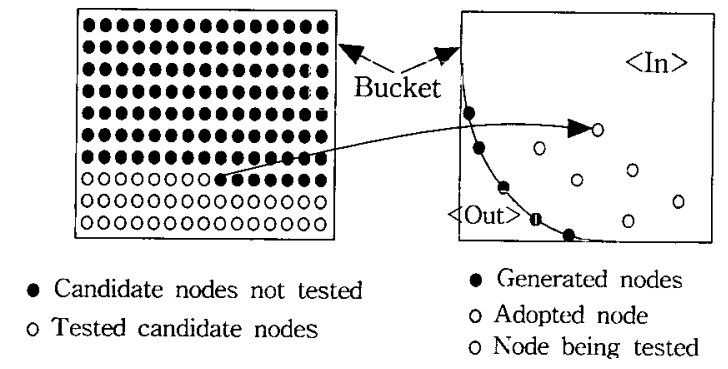

Fig. 5. Schematic view of node generation

\subsection{Element generation}

The Delaunay triangulation method[12] is utilized to generate tetrahedral elements from numerous nodes given in a geometry. The speed of element generation by the Delaunay triangulation method is proportional to the number of nodes. If this method is utilized to generate elements in a geometry with indented shape, elements are inevitably generated even outside the geometry. However, such mis-match elements can be removed by performing the IN/OUT check for gravity center points of such elements. In addition, it is necessary to avoid the generation of those mis-match elements crossing domain boundary by setting node densities on edges to be slightly higher than those inside the domain near the boundaries. The algorithm of element generation mentioned above works well in most cases. However, element shapes obtained are sometimes distorted in a superposed region of several node patterns or near domain boundary. The smooth- ing method called "Laplacian operation"[13] is hear applied to remedy such distorted elements. In this operation, the location of each node is replaced with a mean value of locations of its neighboring nodes.

\subsection{Preparation of input data}

Through the interactive operations mentioned in section 2.2 and 2.3, an user designates material properties and boundary conditions onto the geometry model. Then these are automatically attached on nodes, edges, faces and volume of elements. Such automatic conversion can be performed owing to the special data structure of finite elements such that each part of element knows which geometry part it belongs to. Finally, a complete FE model consisting of mesh, material properties and boundary conditions is obtained.

\section{Examples}

The performance of the system is demonstrated through the mesh generation of several 3D solid structures. Figs. 6(a) and (b) show the examples of mesh generation for strength analysis of an model plane engine. In general, mesh generation time increases in accordance with the increasing number of nodes or the number of total DOFs.

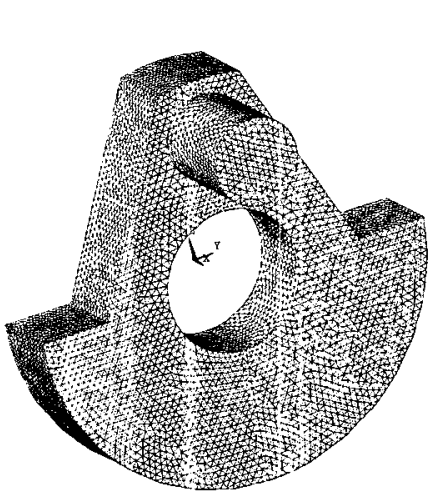

(a) crank shaft

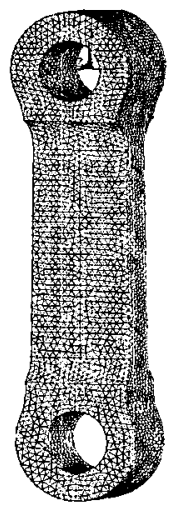

(b) connecting rod
Fig. 6. Examples of mesh generation for strength analysis

To demonstrate actual performances of the present system, the system was applied to the analysis of a model plane engine. As an example, a transient heat conduction problem was analyzed. The temperature of a piston head, as shown in Fig. 7, was initially set $100^{\circ} \mathrm{C}$ throughout. Top face and outer face of the piston head had a temperature of $0^{\circ} \mathrm{C}$. The other faces were insulated. The temperature of the piston head was calculated for subsequent times (0 to $10 \mathrm{secs})$.

In this problem, material properties selected were : thermal conductivity of $1.0 \mathrm{~J} /\left(\mathrm{m} \cdot \mathrm{s} \cdot{ }^{\circ} \mathrm{C}\right)$, specific heat of $1.0 \mathrm{~J} /\left(\mathrm{kg} \cdot{ }^{\circ} \mathrm{C}\right)$ and mass density of $1.0 \mathrm{~kg} / \mathrm{m}^{3}$.

The initial temperature at all nodes was $100^{\circ} \mathrm{C}$. At time $\mathrm{t}=0$, top and outer face have a prescribed temperature of $0^{\circ} \mathrm{C}$; all other surfaces are adiabatic and 
require no data input. A transient solution is performed with 10 uniform time steps of 0.1 seconds each for a total time of 1 second.

Fig. 8 shows a typical FE mesh. The mesh consists of 15,340 tetrahedral quadratic elements and 28,566 nodes. Figs. 9(a) and (b) show a calculated distribut ion of temperature at 0.3 and 1 second, respectively. Fig. 10 shows the measured processing time of a whole process plotted against the total number of nodes. Among the whole process, interactive operations to be done by a node patterns and the assignment of material properties and boundary conditions are performed in about 60 minutes. All the other processes are automatically performed.

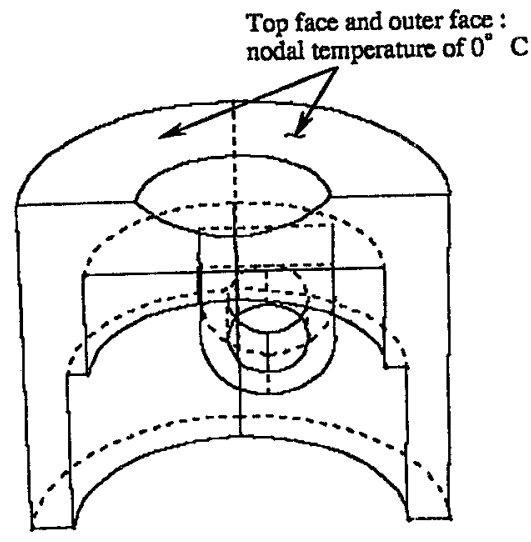

Fig. 7. Boundary condition at time $t=0$

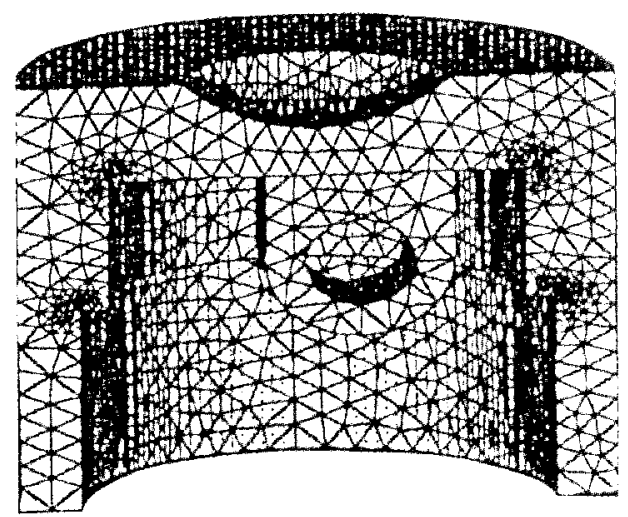

Fig. 8. Mesh for a half of piston head

\section{Conclusions}

An automated FE analysis system for large scale three-dimensional structures was developed. The automatic FE mesh generation technique based on the fuzzy knowledge processing and the computational geometry techniques were integrated in the system, together with one of commercial FE analysis codes and one of commercial solid modelers. Here interactive operations to be done by an user can be performed in a

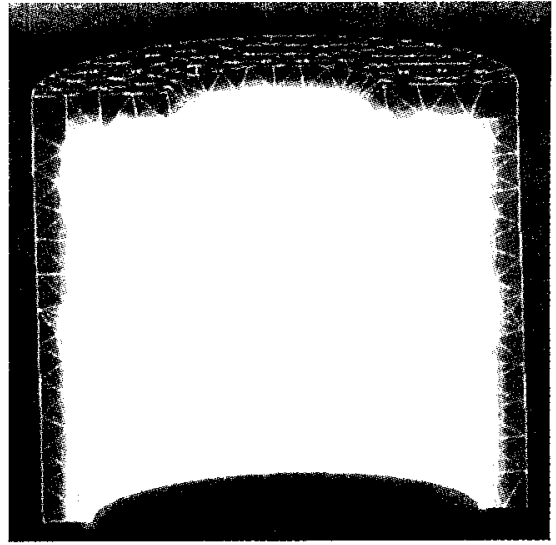

(a) at $0.3 \mathrm{sec}$

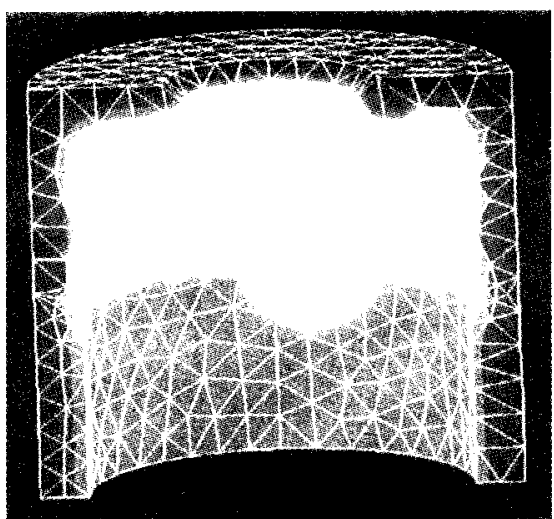

(b) at $1.0 \mathrm{sec}$

Fig. 9. Distribution of temperature

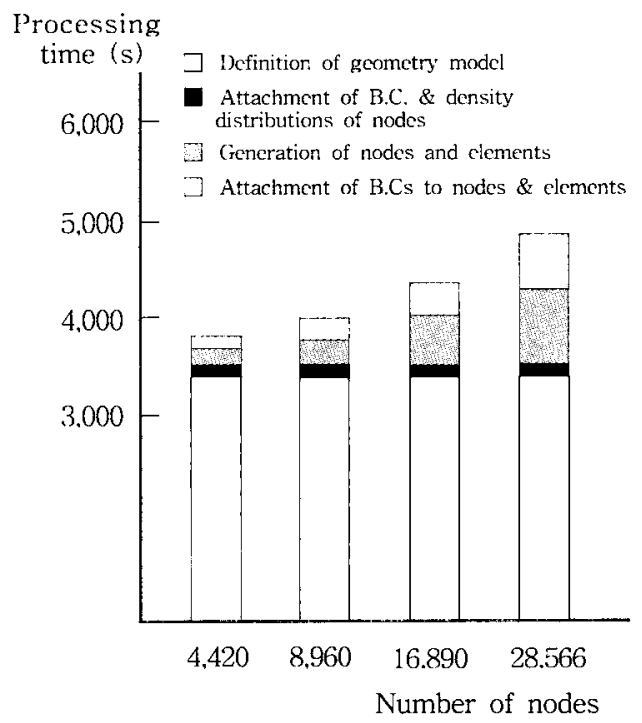

Fig. 10. Processing time vs. number of nodes

reasonably short time even when solving complicated problems of three-dimensional structures. All the other processes which are time consuming and labour-intensive in conventional systems are automati cally performed in a popular personal computer environment. To 
demonstrate practical performances of the present system, the system was used to an analysis of model plane engines such as piston head.

To improve of the present system, thermal fulid simulations using couple phenomenon are currently being carried out.

\section{Acknowledgement}

This research was supported by Research Fund of the Kyonggi University. The authors really appreciate the support.

\section{References}

[1] D.T. Lee et al., "Two Algorithms for Constructing a Delaunay Triangulation", Int. J. of Computer and Information Sciences, vol. 9, pp. 219-242, 1980.

[2] R. Sibson, "Locally Equiangular Triangulations", The Computer Journal, vol. 21, pp. 243-245, 1977.

[3] J.C. Cavendish et al., "An Approach to Automatic Three-Dimensional Finite Element Mesh Generation", Int. J. for Numerical Methods in Eng., vol. 21, pp. 329-347, 1985.

[4] Y. Liu and K. Chen, "A Two-Dimensional Mesh Generator for Variable Order Triangular and Rectangular Elements", Computers and Structures, vol. 29, no. 6, pp. 1033-1053, 1988.

[5] S.H. Lo, "Automatic Mesh Generation and Adaptation by Using Contours", Int. J. for Numerical Methods in Eng., vol. 31, pp. 689-707, 1991.

[6] Joon-Seong Lee et al., "Automatic Mesh Generation for Three-Dimensional Structures Consisting of Free Form Surfaces", Journal of the Korea Society of CAD/CAM Engineers, vol. 1, pp. 65-75, 1996.

[7] Finite Element Analysis, Theory and Application with ANSYS, Prentice Hall, 2000.

[8] H. Chiyokura, Solid Modeling with Designbase : Theory and Implementation, Addition-Wesley, 1988.

[9] L.A. Zadeh, "Fuzzy Algorithms", Information and Control, vol. 12, pp. 94-102, 1968.

[10] L.A. Zadeh, "Outline of a New Approach to the Analysis of Complex Systems and Decision Process", IEEE Transactions on System, Man and Cybernetics, SMC-3, pp. 28-44, 1973.

[11] T. Asano, Practical Use of Bucketing Techniques in Computational Geometry, Computational Geometry, North-Holland, 1985.

[12] D.F. Watson, "Computing the N-Dimensional, Delaunay Tessellation with Application to Voronoi Polytopes", The Computer Journal, vol. 2A, pp. 167-172, 1981.
[13] J.C. Cavendish, "Automatic Triangulation of Arbitrary Planar Domains for the Finite Element Method", Int. J. for Numerical Methods in Eng., Vol. 8, pp. 679-696, 1974.

[14] T. Yamashita et al., "A Simplified Method of Eval-uating Ratcheting in Bellows and a Test of its Validation", Int. J. of Pressure Vessels and Piping, vol. 42, pp. 263-285, 1990.

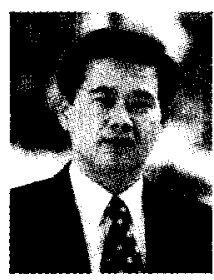

Joon-Seong Lee

He received B.Sc. (1986) and M.Sc. (1988) degrees in Mechanical Engineering from Sung Kyun Kwan University, Suwon, Korea. He worked as a professor during 1988-1991 in the Department of Mechanical Engineering of Korea Military Academy. He received Ph.D. degree in engineering from the University of Tokyo in 1995. Now he is an associate professor in the Division of Electronic \& Mechanical Engineering of Kyonggi University. His current research interest is the development of an intelligent simulation system that will effectively combine numerical simulations such as the finite-element method and soft computing techniques, and its application to various artifacts such as micromachines.

Phone : 031-249-9813

Fax : 031-249-9796

E-mail : jslee1@kuic.kyonggi.ac.kr

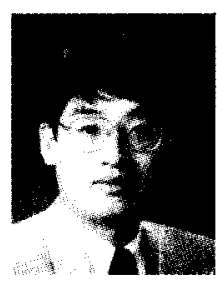

Shin-Pyo Lee

He received B.Sc. (1986), M.Sc. (1988) and Ph. D. (1995) degree in Mechanical Engineering from Seoul National University, Seoul, Korea. Now he is an assistant professor in the Division of Electrical \& Mechanical Engineering of Kyonggi University. His current research interests are thermal system design for nanofluid, developing a capacitive type torque meter and the flow calculation for new ventilation system.

Phone $: 031-249-9814$
Fax $: 031-249-9796$

E-mail : shinpyo@kuic.kyonggi.ac.kr 